\title{
IMPLICIT FUNCTIONS DEFINED BY EQUATIONS WITH VANISHING
}

\section{JACOBIAN *}

BY

\section{GUY ROGER CLEMENTS}

\section{Let}

$$
y_{i}=f_{i}\left(x_{1}, \cdots, x_{n}\right) \quad(i=1,2, \cdots, n),
$$

be $n$ functions, each analytic in the point $\left(x_{1}, \cdots, x_{n}\right)=\left(a_{1}, \cdots, a_{n}\right)$, and let

$$
f_{i}(a)=b_{i} \quad(i=1,2, \cdots, n) .
$$

The nature of the inverse functions

$$
x_{i}=g_{i}\left(y_{1}, \cdots, y_{n}\right)
$$

in the neighborhood of the point $(y)=(b)$ is familiart for the case in which the Jacobian

$$
J=\frac{\partial\left(f_{1}, f_{2}, \cdots, f_{n}\right)}{\partial\left(x_{1}, x_{2}, \cdots, x_{n}\right)}
$$

does not vanish in the point $(x)=(a)$, and for the case in which $J$ vanishes identically in the neighborhood of this point. This paper deals with the intermediate case in which $J$ vanishes in the point $(x)=(a)$, but does not vanish identically in the $n$ variables $x$. Concerning this case, Professor OsGood has pointed out that, as examples show, the solution of the system of equations (1) for $x_{1}, \cdots, x_{n}$ may lead to multiple-valued functions which are not analytic in the point $(y)=(b)$, and has made the suggestion, "Dies dürfte wohl stets der Fall sein." This turns out in fact to be the case whenever an inverse exists throughout the neighborhood of the point $(x)=(a)$.

Some phases of the problem here suggested have been studied by LONGLEY, $\ddagger$ Dederick, $\$$ and URner\| (see also the note to Theorem 4). For the most of their work, $f_{i}$ is a real function of real variables. For the case treated here $f_{i}$

* Presented to the Society, April 28, 1911, and October 26, 1912. See Bulletin, American Mathematical Societ y, vol. 18 (1912), p. 451.

† OsGood, Encyclopädie der Mathematischen Wissenschaften, vol. II, p. 104, Sec. 44.

†Bulletin of the American Mathematical Society, vol. 17 (1910), p. 1.

These Trans a ction s, vol. 14 (1913), p. 143.

|| Ibid., vol. 13 (1912), p. 232. 
is an analytic function of the $n$ complex arguments $x_{1}, x_{2}, \cdots, x_{n}$. For two of the theorems the proof is given only in the case $n=2$.

If $x_{1}, x_{2}, \cdots, x_{n}$ denote $n$ independent complex variables; any region which contains at least the totality of all points $\left(x_{1}, x_{2}, \cdots, x_{n}\right)$ for which

$$
\left|x_{1}-a_{1}\right|<\epsilon, \quad\left|x_{2}-a_{2}\right|<\epsilon, \quad \cdots, \quad\left|x_{n}-a_{n}\right|<\epsilon,
$$

for some $\epsilon>0$, is called a neighborhood of the point $\left(a_{1}, a_{2}, \cdots, a_{n}\right)$. For purposes of simplicity, the neighborhood of the point $(0,0, \cdots, 0)$ is considered throughout this paper.

\section{Part I.}

\section{The Transformat on $\mathrm{T}$.}

Consider the transformation

$T:$

$$
x=f(u, v), \quad y=\varphi(u, v),
$$

(a) $f(u, v)$ and $\varphi(u, v)$ denoting functions of the complex variables $u$ and $v$, single-valued and analytic throughout a neighborhood $R$ of $u=0, v=0$; and satisfying the conditions

$$
\begin{gathered}
f(0,0)=0, \quad \varphi(0,0)=0 ; \\
J(u, v)=f_{u} \varphi_{v}-f_{v} \varphi_{u} \neq 0, \quad J(0,0)=0 .
\end{gathered}
$$

To any point $(u, v)$ of $R$, there corresponds, under $T$, one and but one point $(x, y)$ of the $(x, y)$ space. The totality of points $(x, y)$ which thus correspond to points $(u, v)$ of $R$, constitute a finite region $\bar{R}$ of the $(x, y)$ space. In general, more than one point $(u, v)$ will yield the same pair of values for $x$ and $y$. We shall count such a point $(x, y)$ but once, thus regarding it as completely characterized by its coördinates, and seek an inverse transformation

$$
u=\bar{f}(x, y), \quad v=\bar{\varphi}(x, y)
$$

which will put in evidence all those points $(u, v)$ of $R$ which correspond under $T$ to any point with coördinates $(x, y)$ of $\bar{R}$. Thus the functions $\bar{f}(x, y)$, $\bar{\varphi}(x, y)$ are to be defined only in the points of $\bar{R}$, and there uniquely or as multiple valued functions.

We shall make repeated use of the following form of the Weierstrassian* preparation theorem:

If $F\left(y ; x_{1}, \cdots, x_{n}\right)$ is analytic in the point $(0 ; 0, \cdots, 0)$, and if

* Weierstrass, Abhandlungen aus der Funktionenlehre, p. 105; PiCגRD, Traité d' Analyse, vol. 2, p. 241; Goursat, Cours d'Analyse, vol. 2, Sec. 356, p. 280; Buss, B u 11 e t i n of the American Mathematical Society, vol. 16 (1910), p. 356; Macminlan, ibid., vol. 17 (1910), p. 116. 
$F(0 ; 0, \cdots, 0)=0$; if furthermore $F(y ; 0, \cdots, 0) \neq 0$, and if $y=0$ is a zero of order $m$ of $F(y ; 0, \cdots, 0)$, then $F\left(y ; x_{1}, \cdots, x_{n}\right)$ can be expressed in the form

$$
F\left(y ; x_{1}, \cdots, x_{n}\right) \equiv \Gamma\left(y ; x_{1}, \cdots, x_{n}\right) \omega\left(y ; x_{1}, \cdots, x_{n}\right),
$$

where $\omega\left(y ; x_{1}, \cdots, x_{n}\right)$ is analytic in the point $(0 ; 0, \cdots, 0)$ and $\omega(0 ; 0, \cdots, 0) \neq 0$, and furthermore

$\Gamma\left(y ; x_{1}, \cdots, x_{n}\right) \equiv y^{m}+A_{1}\left(x_{1}, \cdots, x_{n}\right) y^{m-1}+\cdots+A_{m}\left(x_{1}, \cdots, x_{n}\right)$, $A_{i}\left(x_{1}, \cdots, x_{n}\right)$ being analytic in the point $(0, \cdots, 0)$ with $A_{i}(0, \cdots, 0)=0$ $(i=1,2, \cdots, m)$.

We can assume without essential restriction that $f(u, v), \varphi(u, v)$ and $J(u, v)$ satisfy the conditions of this theorem. That the first two conditions are satisfied follows immediately from the hypotheses concerning $T$. But suppose, for example, that $f(u, 0) \equiv 0$. Since $f(u, v)$ is analytic in the point $(0,0)$, it can be written as a series of the form

$$
f(u, v) \equiv f_{1}(u, v)+f_{2}(u, v)+\cdots+f_{i}(u, v)+\cdots,
$$

where $f_{i}(u, v)$ is a homogeneous polynomial of degree $i$ in $u$ and $v$, or vanishes identically. Since by hypothesis, $J(u, v) \neq 0$, it follows that $f(u, v) \neq 0$ and $\varphi(u, v) \neq 0$. Hence there must exist at least one $f_{i}(u, v) \neq 0$. Suppose $j$ to be the degree of the first $f_{i}(u, v)$ which is not identically zero. Make the change of variables

$$
\begin{aligned}
& u=a u^{\prime}+b v^{\prime}, \\
& v=c u^{\prime}+d v^{\prime},
\end{aligned}
$$

subject to the conditions* that

$$
a d-b c \neq 0,
$$

and that the coefficient of $u^{j}$ in the polynomial into which $f_{j}$ is transformed is not zero.

This coefficient, which has the value $f_{j}(a, c)$, is a homogeneous polynomial of degree $j$ in $a$ and $c$ with at least one coefficient different from zero. Taking for $a$ and $c$ any two numbers for which this polynomial does not vanish, one can then always determine $b$ and $d$ so that further $a d-b c \neq 0$. In the same way we see that, to insure $\varphi(u, 0) \neq 0$ and $J(u, 0) \neq 0$, it is sufficient, since $J$ is invariant under linear transformation, $\dagger$ to avoid a finite number of values of the ratio $a: c$.

The transformed functions are in each case single-valued and analytic in the neighborhood of $u^{\prime}=0, v^{\prime}=0$, and vanish in this point. Since the

* Werrrastrass, loc. cit:, p. 113.

† Goursat, Cours d' Analyse, vol. 1, Sec. 29. 
Jacobian determinant for the transformed functions differs from that for the functions $f(u, v)$ and $\varphi(u, v)$ only by the constant factor $a d-b c \neq 0$, the vanishing of the Jacobian in the point $(0,0)$ has not been affected.

Any function

$$
f(u, v) \equiv f_{1}(u, v)+f_{2}(u, v)+\cdots+f_{i}(u, v)+\cdots,
$$

which has a coefficient different from zero for the term in $u$ alone in that first polynomial $f_{i}(u, v)$ which is not identically zero, will be spoken of in the subsequent discussion as normalized.

Theorem 1: The transformation $T$ can never have an inverse

$$
u=\bar{f}(x, y), \quad v=\bar{\varphi}(x, y)
$$

such that both $\bar{f}(x, y)$ and $\bar{\varphi}(x, y)$ are single-valued and analytic throughout the neighborhood $\bar{R}$ of $x=0, y=0$, and vanish when $x=0, y=0$.

Suppose such an inverse to exist. Then $\bar{J}(x, y) \equiv \bar{f}_{x} \bar{\varphi}_{y}-\bar{f}_{y} \bar{\varphi}_{x}$, being a polynomial in the first partial derivatives of functions analytic in $\bar{R}$, is itself analytic in $\bar{R}$. But*

$$
J(u, v) \cdot \bar{J}[f(u, v), \varphi(u, v)] \equiv 1, \text { and } J(0,0)=0 .
$$

From this contradiction follows the truth of the theorem.

Both theorem and proof evidently hold for a similar transformation in any number of variables.

THEOREM 2: If, in the transformation $T, f(u, v)$ and $\varphi(u, v)$ have no common factor in the point $(0,0)$, and if the region $R$ be suitably restricted, then there exists an inverse, defined throughout the neighborhood of $x=0, y=0$, everywhere continuous in that neighborhood, finitely multiple valued, but not single valued, analytic except along a complex one-dimensional locus, and having the value $u=0, v=0$ when $x=0, y=0$.

Suppose the functions $f(u, v)$ and $\varphi(u, v)$ have been normalized and that

$$
\begin{array}{ll}
f_{u}(0,0)=\cdots=f_{u^{k-1}}(0,0)=0, & f_{u^{k}}(0,0) \neq 0 ; \\
\varphi_{u}(0,0)=\cdots=\varphi_{u^{k-1}}(0,0)=0, & \varphi_{u^{k}}(0,0) \neq 0 .
\end{array}
$$

Consider the equations

$$
\begin{aligned}
& f(u, v)-x=0, \\
& \varphi(u, v)-y=0
\end{aligned}
$$

with left members analytic in a region $S$ made up of $R$ for $u$ and $v$, and of the $x$ and $y$ planes.

\footnotetext{
* Jordan, Cours d'Analyse, vol. 1, 2d ed. (1893), p. 89.
} 
From the Weierstrassian preparation theorem, we have

$$
\begin{aligned}
l \quad f(u, v)-x & \equiv\left[u^{k}+A_{1}(v, x) u^{k-1}+\cdots+A_{k}(v, x)\right] a(u, v, x) \\
& \equiv g(u, v, x) a(u, v, x), \\
(5)=[(u, v)-y & \equiv\left[u^{l}+B_{1}(v, y) u^{l-1}+\cdots+B_{l}(v, y)\right] b(u, v, y) \\
& \equiv h(u, v, y) b(u, v, y),
\end{aligned}
$$

where throughout a suitably chosen neighborhood $S^{\prime}$ of the point $(0,0,0,0)$, each of the functions $g, h, a, b$ is analytic, and $a(u, v, x) \neq 0$, $b(u, v, y) \neq 0$. For definiteness, suppose $h_{1}$ so chosen that

$$
|u|<h_{1}, \quad|v|<h_{1}, \quad|x|<h_{1}, \quad|y|<h_{1}, \quad 0<h_{1},
$$

defines such a neighborhood $S^{\prime}$. Then, corresponding to any point $\left(v^{\prime}, x^{\prime}, y^{\prime}\right)$ in a region

$\Sigma: \quad|v|<h_{2}, \quad|x|<h_{2}, \quad|y|<h_{2}, \quad 0<h_{2} \bar{\gtrless} h_{1}$,

the equations $f\left(u, v^{\prime}\right)-x^{\prime}=0, \varphi\left(u, v^{\prime}\right)-y^{\prime}=0$ have respectively exactly $k, l$ roots $u$ for which $|u|<h_{1}$. These are the roots of the polynomials $g\left(u, v^{\prime}, x^{\prime}\right)$ and $h\left(u, v^{\prime}, x^{\prime}\right)$ respectively. Since we seek only those roots $u$ of equations (4) for which $|u|<h_{1}$, when $x$ and $y$ are suitably restricted in absolute value, it therefore will be sufficient to seek the solution of (4) from the equations

$$
\begin{aligned}
& g(u, v, x) \equiv u^{k}+A_{1}(v, x) u^{k-1}+\cdots+A_{k}(v, x)=0, \\
& h(u, v, y) \equiv u^{l}+B_{1}(v, y) u^{l-1}+\cdots+B_{l}(v, y)=0 .
\end{aligned}
$$

Compute for this pair of equations the resultant $R(v, x, y)$. In particular we can make this computation by means of Euclid's algorithm* or Sylvester's determinant. The identical vanishing of $R(v, x, y)$ in $v, x$ and $y$ is the necessary and sufficient condition that $g(u, v, x)$ and $h(u, v, y)$ have a common factor throughout $\Sigma$. But $R(v, 0,0) \neq 0$ and hence, a fortiori, $R(v, x, y) \neq 0$. For if $R(v, 0,0) \equiv 0$, then $g(u, v, 0)$ and $h(u, v, 0)$ must have a common factor in the point $(0,0,0,0)$. Since $a(u, v, 0) \neq 0$, any factor of $f(u, v)$ must be a factor of $g(u, v, 0)$ and vice versa from identities 5. Similarly, any factor of $\varphi(u, v)$ must be a factor of $h(u, v, 0)$ and vice versa. By hypothesis, $f$ and $\varphi$ have no common factor in the point $(0,0)$. Therefore $g(u, v, 0)$ and $h(u, v, 0)$ have no common factor in the point $(0,0,0,0)$ and consequently $R(v, 0,0) \neq 0$.

Since $R .(v, x, y)$ is a polynomial in the variables $A_{i}, B_{j}$ with vanishing constant term, and since the analytic functions $A_{i}, B_{j}(i=1,2, \cdots, k$;

\footnotetext{
* See BOCher's Algebra, Sec. 70, Sec. 76.
} 
$j=1,2, \cdots, l)$ vanish in the point $(0,0)$, it follows that $R(v, x, y)$ is an analytic function of $v, x$ and $y$ in $\Sigma$, which vanishes in the point $(0,0,0)$; that is,

$$
\begin{aligned}
& R(v, x, y) \text { is analytic in } \Sigma ; \\
& R(0,0,0)=0 ; \\
& R(v, 0,0) \neq 0 .
\end{aligned}
$$

Therefore, $R(v, x, y)$ satisfies all the conditions of the Weierstrassian preparation theorem; and if $v=0$ is a zero of order $n$ of $R(v, 0,0)$, then, in a neighborhood

$\Sigma^{\prime}$ :

$$
|v|<h_{3}, \quad|x|<h_{3}, \quad|y|<h_{3}, \quad 0<h_{3} \bar{\gtrless} h_{2},
$$

(7) $R(v, x, y) \equiv\left[v^{n}+C_{1}(x, y) v^{n-1}+\cdots+C_{n}(x, y)\right] c(v, x, y)$

$$
\equiv G(v, x, y) c(v, x, y) \text {, }
$$

where $C_{i}$ is analytic in $(0,0), C_{i}(0,0)=0(i=1,2, \cdots, n)$ and $c(v, x, y)$ is analytic in $(0,0,0)$ and does not vanish there. Further, for any point $\left(x^{\prime}, y^{\prime}\right)$ of a certain region

$\sigma$ :

$$
|x|<h_{4}, \quad|y|<h_{4}, \quad 0<h_{4} \bar{\gtrless} h_{3}
$$

the equation $R\left(v, x^{\prime}, y^{\prime}\right)=0$ has exactly $n$ roots $v$ for which $|v|<h_{3}$, and these are the $n$ roots of the polynomial

$$
v^{n}+C_{1}\left(x^{\prime}, y^{\prime}\right) v^{n-1}+\cdots+C_{n}\left(x^{\prime}, y^{\prime}\right) .
$$

But $v$, thus defined, is an $n$-valued continuous function of the coefficients $C_{1}, \cdots, C_{n}$, and consequently an $n$-valued continuous function of $x$ and $y$ in the point $\left(x^{\prime}, y^{\prime}\right)$.

What is the behavior of this solution as $(x, y)$, starting from $\left(x^{\prime}, y^{\prime}\right)$ is allowed to vary continuously in the region $\sigma$ ?

If $G(v, x, y)$ is reducible, $\mathrm{i}$. e., if

$$
G(v, x, y) \equiv\left[G_{1}(v, x, y)\right]^{l_{1}} \cdots\left[G_{k}(v, x, y)\right]^{l_{k}},
$$

where $G_{i}(v, x, y)$ is analytic in $(0,0,0)$ and $G_{i}(0,0,0)=0(i=1,2$, $\cdots, k)$ then the solution of the equation $G(v, x, y)=0$ evidently can be built up from the solution of the equations

$$
G_{1}(v, x, y)=0, \cdots, G_{k}(v, x, y)=0 .
$$

* Cavchr, Turin Memoirs, 1831; Cooridar, Annals of Mat he matics, second series, vol. 9 (1908), p. 116; Wrber, Lehrbruch der Algebra, Sec. 44; Briot et Bodquer, Theorie des fonctions elliptiques, Chapter 3, p. 31. 
Any $G_{i}(v, x, y)$ can be written in the form

$$
v^{m}+a_{1}(x, y) v^{m-1}+\cdots+a_{m}(x, y),
$$

where $a_{i}(x, y)$ has properties identical with those enumerated above for $C_{i}(x, y)$. Consequently we limit our immediate attention to an equation

$$
M(v, x, y) \equiv v^{m}+a_{1}(x, y) v^{m-1}+\cdots+a_{m}(x, y)=0,
$$

where $M(v, x, y)$ is irreducible, $a_{1}(x, y), \cdots, a_{m}(x, y)$ are analytic in $\sigma$, and $a_{1}(0,0)=0, \cdots, a_{m}(0,0)=0$.

If $m=1$, the equation (8) defines $v$ throughout $\sigma$ as a single-valued and analytic function of $x$ and $y$. If $m>1$, form the discriminant $D(x, y)$ of $M(v, x, y) . D(x, y)$ is a polynomial in $a_{1}(x, y), \cdots, a_{m}(x, y)$ with no constant term, and consequently a single-valued and analytic function of $x$ and $y$ for which $D(0,0)=0$. Since $M(v, x, y)$ is irreducible, $D(x, y) \neq 0$. Each of the equations obtained by setting equal to zero an irreducible factor of $D(x, y)$ defines a continuous locus in the $(x, y)$ space. Corresponding to every point $\left(x_{0}, y_{0}\right)$ for which $D\left(x_{0}, y_{0}\right) \neq 0$, the equation $M\left(v, x_{0}, y_{0}\right)=0$ has $m$ distinct roots, $v_{1}, v_{2}, \cdots, v_{m}$. Consequently $M(v, x, y)=0$ determines $m$ functions $v(x, y)$, each a single-valued and analytic function of $x$ and $y$ throughout a suitably restricted neighborhood of $x=x_{0}, y=y_{0}$, this region in no case extending to a point in which $D(x, y)=0$.

Let $(\bar{x}, \bar{y})$ be any point $(x, y)$ in $\sigma$, for which $D(x, y)=0$. The $m$ roots of the polynomial

$$
M(v, \bar{x}, \bar{y}) \equiv v^{m}+a_{1}(\bar{x}, \bar{y}) v^{m-1}+\cdots+a_{m}(\bar{x}, \bar{y})
$$

are each less than $h_{3}$ in absolute value. Denote them by $\bar{v}_{1}, \bar{v}_{2}, \cdots, \bar{v}_{m}$.

Suppose $\bar{v}_{1}$ is a simple root; then as above, $M(v, x, y)=0$ has a solution $v$, which is an analytic function of $x$ and $y$ in the neighborhood of $x=\bar{x}$, $y=\bar{y}$, and takes the value $\bar{v}_{1}$ in this point. This solution can be extended analytically* at least to any point of $\sigma$ which is not a point of the locus $D(x, y)=0$.

Since $D(\bar{x}, \bar{y})=0$, at least two of the $\bar{v}$ 's are equal. Suppose $\bar{v}_{2}$ is a root of order $k, 1<k \overline{\overline{<}} m$. Give to $\bar{x}$ and $\bar{y}$ increments $\Delta \bar{x}$ and $\Delta \bar{y}$, subject to the single restriction

$$
|\bar{x}+\Delta \bar{x}|<h_{4}, \quad|\bar{y}+\Delta \bar{y}|<h_{4} .
$$

Then $a_{1}(\bar{x}, \bar{y}), \cdots, a_{m}(\bar{x}, \bar{y})$ receive increments $\Delta a_{1}, \cdots, \Delta a_{m}$. Since $a_{i}(x, y)$ is an analytic function of $x$ and $y$, we can so control $\Delta \bar{x}$ and $\Delta \bar{y}$ that $\Delta a_{i}$ shall be arbitrarily small in absolute value. Consequently, if $\epsilon$ be an arbitrarily assigned small positive quantity, it is always possible to

* Encyklopadie der Mathematischen Wissenschaften, vol. II, p. 108, Sec. 47. 
determine a value $\delta$, depending on $\epsilon$, so that if

the equation

$$
\left|\Delta a_{i}\right|<\delta
$$

$(i=1,2, \cdots, m)$

$$
v^{m}+\left[a_{1}(\bar{x}, \bar{y})+\Delta a_{1}\right] v^{m-1}+\cdots+a_{m}(\bar{x}, \bar{y})+\Delta a_{m}=0
$$

has $k$ and only $k$ roots, ${ }^{*} \bar{v}^{\prime}, \bar{v}^{\prime \prime}, \cdots, \bar{v}^{(k)}$, for which

$$
\left|\bar{v}_{2}-\bar{v}^{\prime}\right|<\epsilon, \quad\left|\bar{v}_{2}-\bar{v}^{\prime \prime}\right|<\epsilon, \quad \cdots, \quad\left|\bar{v}_{2}-\bar{v}^{(\dot{k})}\right|<\epsilon .
$$

That is, $k$ of the solutions $v$ can be grouped so as to form a $k$-valued continuous function of $x$ and $y$ in the neighborhood of the point $(\bar{x}, \bar{y})$. If, further, $\Delta \bar{x}$ and $\Delta \bar{y}$ are chosen so that $D(\bar{x}+\Delta \bar{x}, \bar{y}+\Delta \bar{y}) \neq 0$, then, as we have seen, in the neigiborhood of any such point $(\bar{x}+\Delta \bar{x}, \bar{y}+\Delta \bar{y})$, the solutions $v$ can be grouped so as to yield $m$ single-valued and analytic functions of $x$ and $y$.

Now let $\Delta \bar{x}$ and $\Delta \bar{y}$ approach zero while $D(\bar{x}+\Delta \bar{x}, \bar{y}+\Delta \bar{y}) \neq 0$. The inequalities (9) show that there are $k$ and only $k$ of these $m$ roots $v$ which, when $(x, y)$ approaches $(\bar{x}, \bar{y})$, approach as their limiting value $\bar{v}_{2}$, the $k$-fold value of $v$ when $x=\bar{x}, y=\bar{y}$. The other $m-k$ roots can be grouped similarly with regard to $\bar{v}_{1}, \bar{v}_{3}, \cdots, \bar{v}_{m}$. But $(\bar{x}, \bar{y})$ is any point of the locus $D(x, y)=0$. Therefore the roots have this behavior in every point of $D(x, y)=0$. That is, in the neighborhood of any point $(x, y)$ not on $D(x, y)=0$ there are defined $m$ roots $v$, each of which is a single-valued and analytic function of $x$ and $y$ in this point; as $(x, y)$ approaches a point of the locus $D(x, y)=0$, each root remains continuous and has as its limiting value some one of the roots at that point. Therefore $M(v, x, y)=0$ defines $v$ throughout $\sigma$ as an $m$-valued, continuous function of $x$ and $y$, analytic, with $m$ distinct branches, except in points of the complex one-dimensional locus $D(x, y)=0$, where it is continuous and less than $m$ valued. Since $M(v, 0,0)=0$ has an $m$-tuple root $v=0$, every element of this $m$-fold continuous function has the value zero when $x=0, y=0$.

This discussion is typical for any of the equations $G_{1}^{\prime}(v, x, y)=0, \cdots$, $G_{k}(v, x, y)=0$. Let $h$ denote the smallest of the $k$ numbers $h_{4}$, and $H$ the largest of the $h_{3}$. Then, throughout the region

$\sigma^{\prime}$ :

the equation

$$
|x|<h, \quad|y|<h,
$$

$$
G(v, x, y) \equiv\left[G_{1}(v, x, y)\right]^{l_{1}} \cdots\left[G_{k}(v, x, y)\right]^{l_{k}}=0
$$

defines $v$ as a function of $x$ and $y$ for which $|v|<H$, and which moreover is

* See the reference above to CAUCHY and others. See Nedmann, Abelsche Integrale, 2d ed., (1884), p. 125, for a definition of continuity for multiple-valued functions. 
everywhere continuous, not more than $n$-valued, analytic except along a complex one-dimensional locus, and has the value zero when $x=0, y=0$. If $G_{i}$ is a polynomial in $v$ of degree $m_{i}$, then $n=\sum_{i=1}^{k} l_{i} m_{i}$.

We saw above that in the neighborhood of the origin, this equation $G(v, x, y)=0$ solves completely the equation $R(v, x, y)=0$. That is, if we choose arbitrarily a point $\left(x^{\prime}, y^{\prime}\right)$ in $\sigma^{\prime}$, there will always be at least one point $\left(v, x^{\prime}, y^{\prime}\right)$ in $\Sigma^{\prime}$ for which $R\left(v, x^{\prime}, y^{\prime}\right)=0$. But in every point ( $v, x, y)$ of $\Sigma^{\prime}$ for which $R(v, x, y)=0$, the left members of (4), which when the coördinates of this point are substituted become functions of $u$ alone, have a common factor, thus defining for $u$ a value or values in the point $\left(x^{\prime}, y^{\prime}\right)$.

Since $u$ and $v$ enter symmetrically in the foregoing discussion, the results apply when $u$ and $v$ are interchanged. Thus, without making use of any assumption concerning the value of $J(u, v)$ in $R$, we have shown the existence of functions $u(x, y)$ and $v(x, y)$ which in the region $\sigma^{\prime}$ fulfill the demands of the theorem, with a single possible exception.

May this inverse not be single-valued for some transformation $T$ satisfying the hypotheses of Theorem 2 ? If the inverse is single-valued, $v$ must be a single-valued function of $x$ and $y$; hence in the equation (8) which defines $v$, $m$ must have the value one. But if $m=1, v$ is a single-valued and analytic function of $x$ and $y$ throughout the neighborhood of $x=0, y=0$. Similarly, if the inverse is single-valued $u$ must be a single-valued and analytic function of $x$ and $y$ throughout the neighborhood of $x=0, y=0$. Both $u(x, y)$ and $v(x, y)$ have the value zero when $x=0, y=0$. This is in direct contradiction to Theorem 1 . Hence Theorem 2 is proved.

If instead of the transformation $T$ we consider the transformation $T^{\prime \prime}$, whose definition differs from that for $T$ in that it omits the condition $(c)$, we deduce the following theorem:

Theorem 3: If to each point $(x, y)$ of the region $\bar{R}$ there corresponds in consequence of $T^{\prime}$ a finite number of points $(u, v)$ in a suitably restricted region $R$, and if to some point $(x, y)$ of $\bar{R}$ there corresponds more than one point $(u, v)$ of $R$, then there exists an inverse, defined throughout the neighborhood of $x=0$, $y=0$, everywhere continuous in that neighborhood, finitely multiple-valued, but not single-valued, analytic except along a complex one-dimensional locus, and having the value $u=0, v=0$ when $x=0, y=0$.

If to each point of the region $\bar{R}$ there corresponds in consequence of $T^{\prime}$ a single point $(u, v)$ of the region $R$, then $u$ and $v$ are analytic in $x$ and $y$ in the point $x=0, y=0$.

The functions $f(u, v)$ and $\varphi(u, v)$ can have no common factor in the point $(0,0)$, for if they did, the locus defined by equating to zero this common factor 
would correspond to the point $x=0, y=0$, contrary to the hypothesis that to each point $(x, y)$ of $\bar{R}$ there correspond only a finite number of points $(u, v)$ of $R$. In particular, neither $f(u, v)$ nor $\varphi(u, v)$ can be identically zero.

We show next, that because of this condition that to each point $(x, y)$ there shall correspond a finite number of points $(u, v)$ it follows that $J(u, v) \neq 0$. Since $f(u, v)$ is not a constant, there must be a point $\left(u_{1}, v_{1}\right)$ in $R$ at which a first partial derivative of $f(u, v)$ with respect to one of its arguments, say $f_{u}(u, v)$, is not zero. If $J(u, v) \equiv 0$, then in a suitable neighborhood of $x=x_{1}=f\left(u_{1}, v_{1}\right), y$ is expressible as an analytic function of $x$ alone.* Consequently the same point $(x, y)$ will correspond to every point $(u, v)$ which is a point of the locus $f(u, v)=x_{1}$ and is within a suitable neighborhood $S$ of $\left(u_{1}, v_{1}\right)$ in which $f_{u}(u, v) \neq 0$. This would set up a one-to-infinity correspondence, contrary to hypothesis. Therefore $J(u, v) \neq 0$.

Two cases must be considered: $J(0,0)=0$, and $J(0,0) \neq 0$. If $J(0,0)=0$, all the conditions of Theorem 2 are fulfilled. Consequently the inverse must be multiple-valued and must satisfy all the conclusions of the first paragraph of Theorem 3 . If $J(0,0) \neq 0$, the transformation $T^{\prime}$ defines $u$ and $v$ as analytic in $x$ and $y$ in the point $(0,0)$ and sets up a one-to-one relation between suitably chosen neighborhoods of the point $(x, y)=(0,0)$ and the point $(u, v)=(0,0)$. Hence the theorem is proved.

It follows from Theorem 1 and from Theorem 3 that there can not exist a transformation $T$ for which the inverse is single-valued throughout any neighborhood $\bar{R}$ of the point $x=0, y=0$.

\section{Part II.}

\section{More General Systems of Equations.}

\section{TheOREM 4:† The system of equations}

* Jordan, Cours d'Analyse, Vol. I, 2d ed. (1893), p. 86.

$\dagger$ This theorem is a generalization of the Weierstrass preparation theorem. It was developed without knowledge of the important results published in these $\mathrm{T}$ ra n s a c t i o $\mathbf{n}$ s for April, 1912 , by G. A. Busss under the title $A$ generalization of Weierstrass's preparation theorem for power series in several variables, and it differs from the principal theorem of that paper in its hypotheses and consequently in the scope of its application.

Professor Busss considers certain " characteristic" polynomials, $g_{m}\left(y_{1}, \cdots, y_{p}\right)$, namely the homogeneous polynomials of lowest degree in the series $f_{m}\left(0, \cdots, 0 ; y_{1}, \cdots, y_{p}\right)$, and discusses the solution of such a set of equations as we are considering under the hypothesis that the resultant $R$ of these polynomials $g_{m}$ be different from zero. This is a condition sufficient to establish results with which, except in a simple case, the results of my Theorem 4 are identical. My hypotheses impose no condition on the value of $R$, but my condition $J_{k}(0, \cdots, 0 ; 0, \cdots, 0)+0$, demands that $p-1$ of the characteristic polynomials $g_{m}$ be linear and that at least one determinant of their matrix be different from zero. It was erroneously assumed by Dr. L. L. Dines in a paper presented to the American Mathematical Suciety on October 26, 1912 (see Bulletin, vol. 19, p. 165) that I had required in all caees $R+0$. In the case $R+0$, my theorem is a special case of that announced by Professor Burss. 
where

$$
\begin{gathered}
f_{1}\left(x_{1}, \cdots, x_{n} ; y_{1}, \cdots, y_{p}\right)=0 \\
f_{2}\left(x_{1}, \cdots, x_{n} ; y_{1}, \cdots, y_{p}\right)=0, \\
\cdot \cdot \cdot \cdot \cdot \cdot \cdot \cdot \cdot \cdot \\
f_{p}\left(x_{1}, \cdots, x_{n} ; y_{1}, \cdots, y_{p}\right)=0,
\end{gathered}
$$

(a) $f_{i}(i=1,2, \cdots, p)$ is an analytic function of its arguments throughout a neighborhood $R$ of $(x)=0,(y)=0$;

$$
\begin{gathered}
f_{i}(0, \cdots, 0 ; 0, \cdots, 0)=0 ; \\
J_{1} \equiv \frac{\partial\left(f_{1}, f_{2}, \cdots, f_{p}\right)}{\partial\left(y_{1}, y_{2}, \cdots, y_{p}\right)}=0 \quad \text { when }(x)=0,(y)=0, \\
\cdot \cdot \cdot \cdot \cdot \cdot \cdot \cdot \cdot \cdot \cdot \quad \text { when }(x)=0,(y)=0, \\
J_{k-1} \equiv \frac{\partial\left(J_{k-2}, f_{2}, \cdots, f_{p}\right)}{\partial\left(y_{1}, y_{2}, \cdots, y_{p}\right)}=0 \quad \text { when }(x)=0,(y)=0, \\
J_{k} \equiv \frac{\partial\left(J_{k-1}, f_{2}, \cdots, f_{p}\right)}{\partial\left(y_{1}, y_{2}, \cdots, y_{p}\right)} \neq 0 \quad \text {. }
\end{gathered}
$$

defines $y_{1}, y_{2}, \cdots, y_{p}$ as functions of $x_{1}, x_{2}, \cdots, x_{n}$, continuous throughout the neighborhood of $(x)=0$. With a suitable counting of multiplicities always present this solution is $k$-fold; it is analytic with the possible exception of complex $(n-1)$-dimensional loci where the roots, in general distinct, become coincident. When $(x)$ is zero, $(y)$ is zero.

If $p=1$, this theorem is the Weierstrassian preparation theorem; for the conditions then become $f_{1}\left(x_{1}, \cdots, x_{n} ; y_{1}\right)=0$, where $f_{1}(0, \cdots, 0 ; 0)=0$; $f_{1 y_{1}}(0, \cdots, 0 ; 0)=\cdots=f_{1 y_{1} k-1}(0, \cdots, 0 ; 0)=0 ; f_{1 y_{1}^{k}}(0, \cdots, 0 ; 0) \neq 0$. Hence, throughout a complete neighborhood of the point $(0, \cdots, 0)$, the equation $f_{1}\left(x_{1}, \cdots, x_{n} ; y_{1}\right)=0$ defines $y_{1}$ as a function of $x_{1}, \cdots, x_{n}$, either analytic throughout the region, or everywhere continuous and analytic except along certain loci where the roots, in general distinct, become coincident. If we count multiple roots in the usual way, this solution is $k$-valued.

On the other hand, when the restriction is made that $f_{2}, \cdots, f_{p}$ have linear characteristic polynomials, the results of Professor Burss' theorem follow from mine. Thus the theorems overlap while neither is a special case of the other.

At the end of Theorem 4, I give two examples neither of which satisfies the condition $R \neq 0$, for " if the characteristic polynomials are all of degree one, their determinant is the resultant $R^{\prime \prime}$ (Bliss, loc. cit., p. 136), and in each case this determinant is readily seen to be zero. An example satisfying my Theorem 5 , but one for which $R=0$, is

$$
\begin{gathered}
y_{2}+y_{1} y_{3}+y_{1}^{3}-x_{1}=0, \\
y_{2}-x_{2}=0, \quad y_{3}-x_{3}=0 ;
\end{gathered}
$$

for which $J_{1}=y_{3}+3 y_{1}^{2}, J_{2}=6 y_{1}, J_{3}=6$. 
The condition $J_{k}(0, \cdots, 0 ; 0, \cdots, 0) \neq 0$ requires that at least one determinant from the matrix

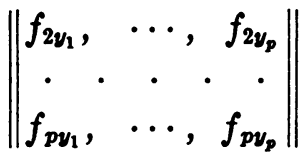

shall be different from zero when $(x)=0,(y)=0$. For definiteness suppose the notation so chosen that

$$
\frac{\partial\left(f_{2}, \cdots, f_{p}\right)}{\partial\left(y_{2}, \cdots, y_{p}\right)} \neq 0
$$

when $(x)=0,(y)=0$. Then the last row of this determinant,

$$
f_{p y_{z}}, \cdots, f_{p y_{p}},
$$

must have at least one element which is not zero at the origin. Suppose the notation so chosen that

$$
f_{p y_{p}}(0, \cdots, 0 ; 0, \cdots, 0) \neq 0 .
$$

Then we can write

$$
\begin{aligned}
& f_{p}\left(x_{1}, \cdots, x_{n} ; y_{1}, \cdots, y_{p}\right) \\
& \quad \equiv\left[y_{p}-A\left(x_{1}, \cdots, x_{n} ; y_{1}, \cdots, y_{p-1}\right)\right] K\left(x_{1}, \cdots, x_{n} ; y_{1}, \cdots, y_{p}\right),
\end{aligned}
$$

where $K \neq 0$ in a suitable neighborhood of the origin.

Replacing $y_{p}$ by $A\left(x_{1}, \cdots, x_{n} ; y_{1}, \cdots, y_{p-1}\right)$ in all the equations of the set (10), we are led to the case of $p-1$ equations

$$
f_{1}\left(x_{1}, \cdots, x_{n} ; y_{1}, \cdots, y_{p-1}, A\right)=F_{1}\left(x_{1}, \cdots, x_{n} ; y_{1}, \cdots, y_{p-1}\right)=0 \text {, }
$$

$$
\begin{aligned}
& f_{p-1}\left(x_{1}, \cdots, x_{n} ; y_{1}, \cdots, y_{p-1}, A\right)=F_{p-1}\left(x_{1}, \cdots, x_{n} ; y_{1}, \cdots, y_{p-1}\right)=0, \\
& f_{p}\left(x_{1}, \cdots, x_{n} ; y_{1}, \cdots, y_{p-1}, A\right) \equiv 0,
\end{aligned}
$$

which obviously satisfy conditions $(a)$ and $(b)$ of the theorem. We show that the new Jacobian determinant

- $\bar{J} \equiv\left|F_{1 y_{1}} F_{2 y_{2}} \cdots F_{p-1, y_{p-1}}\right|$

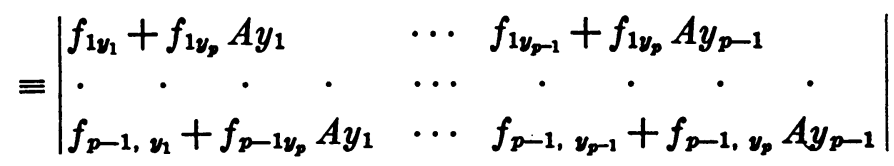

where $y_{p}$ is to be replaced by $A$, is zero at the origin, and that the successive $\bar{J}_{i}$, formed from this new determinant $\bar{J}$, satisfy conditions (c) of the theorem.

First, since $f_{p} \equiv\left(y_{p}-A\right) K, J$ has the form 


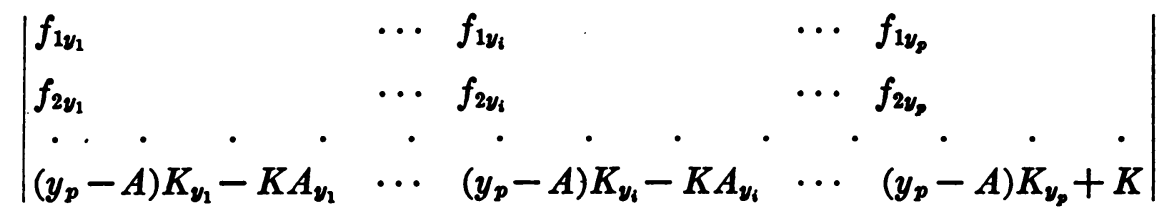

which is equal to

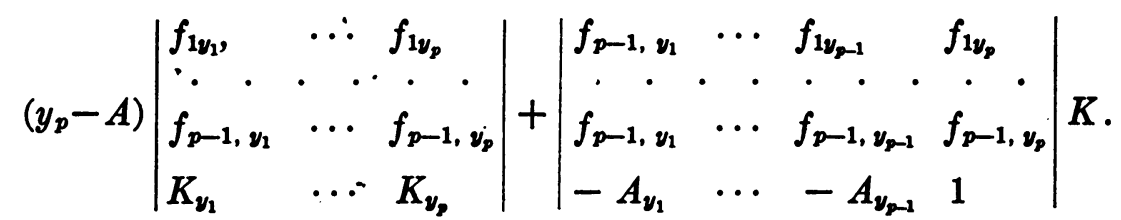

Set

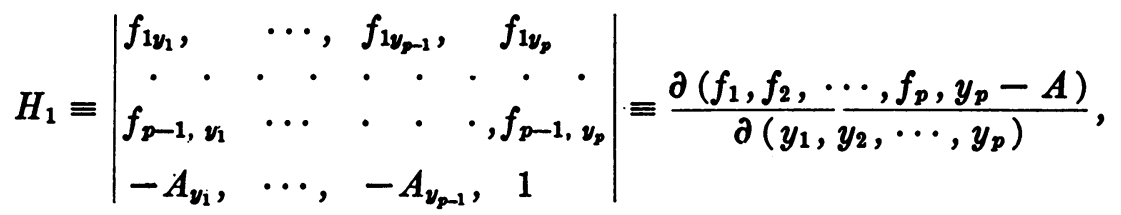

and

$$
H_{i+1} \equiv \frac{\partial\left(H_{i}, f_{2}, \cdots, f_{p-1}, y_{p}-A\right)}{\partial\left(y_{1}, y_{2}, \cdots, y_{p-1}, y_{p}\right)}
$$

In the determinant $H_{1}$ multiply the elements of the last column by the lowest element of the $i$ th column, and subtract from the $i$ th column $(i=1,2, \cdots$, $p-1)$. Then

$$
H_{1} \equiv\left|\begin{array}{ccccc}
f_{1 y_{1}}+f_{1 y_{p}} A_{y_{1}}, & \cdots, & f_{1 y_{p-1}} & +f_{1 y_{p}} A_{y_{p-1}} \\
\cdot & \bullet & \cdot & \cdot & \cdot \\
f_{p-1, y_{1}}+f_{p-1, y_{p}} A_{y_{1}}, & \cdots, & f_{p-1, y_{p-1}}+f_{p-1, y_{p}} A_{y_{p-1}}
\end{array}\right|
$$

That is, $H_{1}$ is the function which under the substitution $y_{p}=A$ reduces to the Jacobian $\bar{J}$ for the $(p-1)$ equations (15). Consequently $H_{i+1}$ goes over into $\bar{J}_{i+1}$ under the same substitution. We have seen that when we replace $f_{p}$ by $\left(y_{p}-A\right) K$,

$$
J=\left(y_{p}-A\right)\left|f_{1 y} \cdots f_{p-1, y_{p-1}} K_{y_{p}}\right|+K H_{1} \text {. }
$$

Suppose it has been shown that

$$
J_{n}=\left(y_{p}-A\right) L+M H_{1}+N H_{2} \cdots+Q H_{n-1}+K^{n} H_{n} .
$$

We will now show that for any $n$

$$
J_{n+1} \equiv\left(y_{p}-A\right) L^{\prime}+M^{\prime} H_{1}+\cdots+Q^{\prime} H_{n}+K^{n+1} H_{n+1} \text {, }
$$

where $L, M, \cdots, L^{\prime}, M^{\prime}, \cdots$ denote analytic functions of $(x)$ and $(y)$ whose particular form is of no interest. By definition, 


$$
J_{n+1} \equiv\left|\begin{array}{lll}
J_{n y_{1}} & \cdots & J_{n y_{p}} \\
f_{2 y} & \cdots & \\
\cdots & \cdots & f_{p-1, y_{p}} \\
f_{p y_{1}} & \cdots & f_{p y_{p}}
\end{array}\right|
$$

To replace $f_{p}$ by $\left(y_{p}-A\right) K$ affects only the first and last rows. If we leave the first row unchanged for the moment, and replace $f_{p}$ by $\left(y_{p}-A\right) K$ in the last row, we have

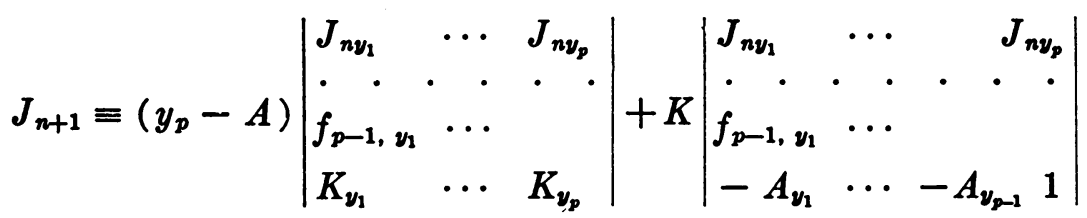

We lay aside the first term, which admits $y_{p}-A$ as a factor, and in the second determinant replace $J_{n}$ by its value from (19). The elements of the first row will be

$$
\begin{array}{r}
\left(y_{p}-A\right) L_{y_{i}}-L A_{y_{i}}+H_{1} M_{y_{i}}+H_{1 y_{i}} M+\cdots+n K^{n-1} K_{y_{i}} H_{n}+K^{n} H_{n y_{i}} \\
\left(i=1,2, \cdots, p, \text { where } A_{y_{p}} \text { is to be replaced by }-1\right) .
\end{array}
$$

Expand this determinant in terms of the minors of its first row, then regroup into the sum of the $2 n+2$ determinants of order $p$

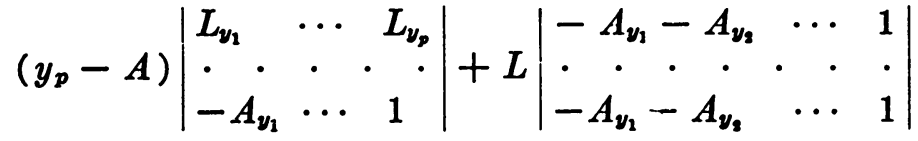

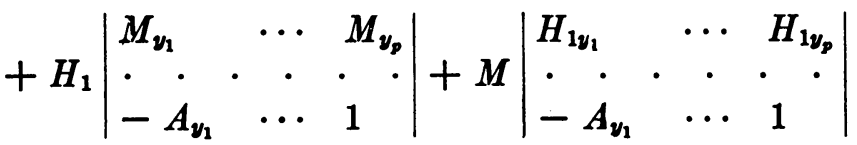

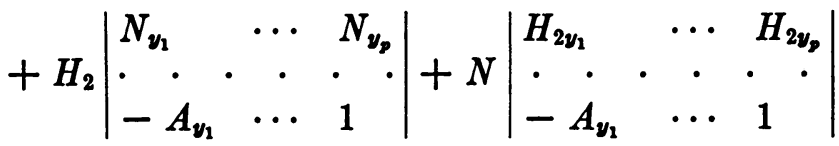

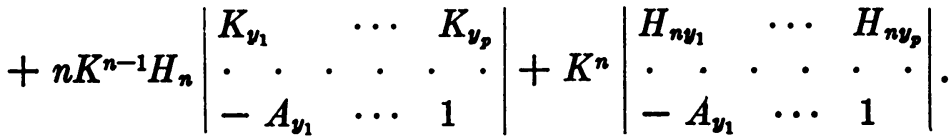

Of the determinants in the right-hand column, the first is identically zero, the second is $H_{2}$, the third is $H_{3}, \cdots$, the $(n+1)$ st is $H_{n+1}$. Therefore, if the identity (19) is true, then also is

$$
J_{n+1} \equiv\left(y_{p}-A\right) L^{\prime}+M^{\prime} H_{1}+N^{\prime} H_{2}+\cdots+Q^{\prime} H_{n}+K^{n+1} H_{n+1} .
$$


But (18a) establishes this relation for $n=0$; it is therefore true for all values of $n$.

Since $J_{1}, \cdots, J_{k-1}$, and $y_{p}-A$ are zero at the origin, and since $K \neq 0$, $J_{k} \neq 0$ at that point, it follows from these $k$ identities considered for the values $n=0, \cdots, n=k-1$ successively, that $H_{1}=0, H_{2}=0, \cdots, H_{k-1}=0$, $H_{k} \neq 0$, when $(x)=0,(y)=0$. Consequently the $k$ functions $\bar{J}_{1}, \bar{J}_{2}$, $\cdots, \bar{J}_{k}$ into which the substitution $y_{p}=A$ carries the $H$ 's satisfy the conditions

when

$$
\bar{J}_{1}=0, \quad \bar{J}_{2}=0, \cdots, \bar{J}_{k-1}=0, \bar{J}_{k} \neq 0,
$$

$$
(x)=0, y_{1}=0, \cdots, y_{p-1}=0 .
$$

This process depends in no essential way on the number $p$ of the theorem. We have rather shown that from any system of equations $(p>1)$ satisfying the hypotheses of Theorem 4, it is always possible to build up a second system, one less in number, satisfying exactly analogous hypotheses. Consequently, if we carry through this process $p-1$ times, we replace the set of equations (10) by the new set

$$
\begin{aligned}
y_{p} & =A_{0}\left(y_{1}, \cdots, y_{p-1} ; x_{1}, \cdots, x_{n}\right), \\
y_{p-1} & =A_{1}\left(y_{1}, \cdots, y_{p-2} ; x_{1}, \cdots, x_{n}\right), \\
& \cdot \cdot \cdot \cdot \cdot \cdot \cdot \cdot \\
y_{3} & =A_{p-3}\left(y_{1}, y_{2} ; x_{1}, \cdots, x_{n}\right), \\
y_{2} & =A_{p-2}\left(y_{1} ; x_{1}, \cdots, x_{n}\right), \\
0 & =F\left(y_{1} ; x_{1}, \cdots, x_{n}\right),
\end{aligned}
$$

where $A_{m}(m=0,1, \cdots, p-2)$ and $F$ are single-valued and analytic functions of their arguments in the origin and vanish there, and where further $F_{y_{1}}(0 ; 0, \cdots, 0)=\cdots=F_{y_{1}^{k-1}}(0 ; 0, \cdots, 0)=0, \quad F_{y^{k}}(0 ; 0, \cdots, 0) \neq 0$. In $A_{p-8}$ replace $y_{2}$ by $A_{p-2}\left(y_{1} ; x_{1}, \cdots, x_{n}\right)$; then in $A_{p-4}$ replace $y_{2}$ and $y_{3}$ by their values in $y_{1}$ and $(x)$, and so on to $A_{0}$. The system (21) is thus replaced by the equivalent simpler system

$$
\begin{aligned}
& y_{2}=A_{p-2}\left(y_{1} ; x_{1}, \cdots, x_{n}\right) \quad=a_{2}\left(y_{1} ; x_{1}, \cdots, x_{n}\right), \\
& y_{3}=A_{p-3}\left(y_{1}, a_{2} ; x_{1}, \cdots, x_{n}\right) \quad=a_{3}\left(y_{1} ; x_{1}, \cdots, x_{n}\right) \text {, } \\
& y_{p-1}=A_{1}\left(y_{1}, a_{2}, \cdots, a_{p-2} ; x_{1}, \cdots, x_{n}\right)=a_{p-1}\left(y_{1} ; x_{1}, \cdots, x_{n}\right), \\
& y_{p}=A_{0}\left(y_{1}, a_{2}, \cdots, a_{p-1} ; x_{1}, \cdots, x_{n}\right)=a_{p}\left(y_{1} ; x_{1}, \cdots, x_{n}\right), \\
& F\left(y_{1} ; x_{1}, \cdots, x_{n}\right)=0 \text {. }
\end{aligned}
$$


The existence and the uniqueness of the equations $y_{m}=a_{m}(m=2, \cdots, p)$ is a direct consequence* of condition (12), but the method we have followed further establishes a set of working conditions on the function $F\left(y_{1} ; x_{1}, \cdots, x_{n}\right)$. If we replace $F$ by the equivalent Weierstrassian polynomial of degree $k$ in $y_{1}$, then $y_{1}$ is defined by the equation

$$
\begin{aligned}
r\left(y_{1} ; x_{1}, \cdots, x_{n}\right) \equiv y_{1}^{k}+S_{1}\left(x_{1}, \cdots, x_{n}\right) y_{1}^{k-1} & +\cdots \\
& +S_{k}\left(x_{1}, \cdots, x_{n}\right)=0 .
\end{aligned}
$$

In every point of the region of definition which is not a point of the locus defined by equating to zero the discriminant of some irreducible factor of $r\left(y_{1} ; x_{1}, \cdots, x_{n}\right)$, each of the roots $y_{1}$ of the equation $r=0$ can be expressed as a single-valued and analytic function of $x_{1}, \cdots, x_{n}$, and hence by an immediate substitution $y_{2}, \cdots, y_{p}$ are determined to be single-valued and analytic in $x_{1}, \cdots, x_{n}$ in such a point. If we count possible multiple roots in the usual way, the solution is $k$-valued. If in the point $(0 ; 0, \cdots, 0)$, $r\left(y_{1} ; x_{1}, \cdots, x_{n}\right)$ is completely reducible into factors linear in $y_{1}$, the solution is analytic throughout the neighborhond of this point; but in general $r$ is not thus reducible! We have, however, discussed in detail the character of $y_{1}$ as defined by means of equation (23). It 'follows further, from a theorem due to Poincare, $\dagger$ that $y_{m}$ must also satisfy an equation

$y_{m}^{k}+Q_{m 1}\left(x_{1}, \cdots, x_{n}\right) y_{m}^{k-1}+\cdots+Q_{m k}\left(x_{1}, \cdots, x_{m}\right)=0 \quad(m=2,3, \cdots, p)$, where $Q_{m i}\left(x_{1}, \cdots, x_{n}\right)(m=2, \cdots, p ; i=1, \cdots, k)$ is analytic in the point $\left(x_{1}, \cdots, x_{n}\right)=(0, \cdots, 0)$ and vanishes there. Therefore throughout a suitable neighborhood of $\left(x_{1}, \cdots, x_{n}\right)=(0, \cdots, 0), y_{m}$ is defined as a continuous function of $x_{1}, \cdots, x_{n}$, analytic with the possible exception of a complex $(n-1)$-dimensional locus, defined by equating to zero the discriminant of some irreducible factor of $r\left(y_{1} ; x_{1}, \cdots, x_{n}\right)$, where roots elsewhere distinct become coincident. Furthermore $y_{m}=0$ when $\left(x_{1}, \cdots, x_{n}\right)$ $=(0, \cdots, 0)(m=1,2, \cdots, p)$. Hence the theorem is proved.

A simple example of a set of equations satisfying the conditions of this theorem and having solutions everywhere analytic is the system

for which

$$
\begin{aligned}
y_{1}^{2}-y_{2}-2 y_{3}-y_{4}=0, & y_{2}-x_{1}^{2}=0, \\
y_{3}-x_{1} x_{2}=0, & y_{4}-x_{2}^{2}=0 ;
\end{aligned}
$$

The solution is

$$
J_{1} \equiv 2 y_{1}, \quad J_{2} \equiv 2 .
$$

$$
y_{1}=x_{1}+x_{2} \quad \text { or } \quad y_{1}=-x_{1}-x_{2}
$$

* Picard, Traité d'Analyse, 2d ed. (1905), vol. 2, p. 267.

† Poincart, Thesis, Paris, 1879, p. 13, Cor. II. 
with the functions

$$
y_{2}=x_{1}^{2}, \quad y_{3}=x_{1} x_{2}, \quad y_{4}=x_{2}^{2} \text {. }
$$

A second example is the system

$$
\begin{gathered}
y_{1}^{2}+y_{2}+2 y_{3}+y_{4}-2 x_{1} y_{1}-2 x_{2} y_{1}=0, \\
y_{2}-x_{1}^{2}=0, \quad y_{3}-x_{1} x_{2}=0, \quad y_{4}-x_{2}^{2}=0,
\end{gathered}
$$

for which $J_{1} \equiv 2\left(y_{1}-x_{1}-x_{2}\right), J_{2} \equiv 2$. The inverse is given by the system of functions

$$
y_{1}=x_{1}+x_{2}, \quad y_{2}=x_{1}^{2}, \quad y_{3}=x_{1} x_{2}, \quad y_{4}=x_{2}^{2},
$$

counted twice.

Note that in the problems as set, every equation has as its left member an irreducible function of its arguments.

The following theorem gives an example of a further and more general type of problem.

THEOREM 5: The transformation

where

$$
\begin{aligned}
& x_{1}=f_{1}\left(y_{1}, \cdots, y_{n}\right), \\
& x_{2}=f_{2}\left(y_{1}, \cdots, y_{n}\right), \\
& \cdot \cdot \cdot \cdot \cdot \cdot \\
& x_{n}=f_{n}\left(y_{1}, \cdots, y_{n}\right),
\end{aligned}
$$

(a) $f_{i}$ is a single valued and analytic function of $y_{1}, \cdots, y_{n}$ in the point $(0, \cdots, 0)$ and vanishes there $(i=1,2, \cdots, n)$;

$$
\begin{aligned}
J_{1}(0, \cdots, 0) & =\left.\frac{\partial\left(f_{1}, \cdots, f_{n}\right)}{\partial\left(y_{1}, \cdots, y_{n}\right)}\right|_{(y)=0}=0, \\
J_{k-1}(0, \cdots, 0) & =\left.\frac{\partial\left(J_{k-2}, f_{2}, \cdots, f_{n}\right)}{\partial\left(y_{1}, \cdots, y_{n}\right)}\right|_{(y)=0}=0, \\
J_{k}(0, \cdots, 0) & =\left.\frac{\partial\left(J_{k-1}, f_{2}, \cdots, f_{n}\right)}{\partial\left(y_{1}, \cdots, y_{n}\right)}\right|_{(y)=0} 0
\end{aligned}
$$

has a $k$ valued continuous inverse, defined throughout the neighborhood of $(x)=0$. This inverse is analytic, with $k$ distinct branches, except along a complex $(n-1)$-dimensional locus, where it is continuous and less than $k$ valued. Finally $(y)=0$ when $(x)=0$.

If we consider the set of equations

$$
f_{1}\left(y_{1}, \cdots, y_{n}\right)-x_{1}=0, \cdots, \quad f_{n}\left(y_{1}, \cdots, y_{n}\right)-x_{n}=0,
$$

in an arbitrary complete neighborhood of the point $(x)=0,(y)=0$, which 
includes the region defined for the transformation (24), this theorem follows at once from Theorem 4, with the exception of the requirement that the branches of the inverse shall be distinct in any point in which they are analytic. That the inverse is defined in all points of a complete neighborhood $\bar{R}$ of $(x)=0$, follows as in Theorem 2. To see that in general no two branches of this solution are the same at a point of $\vec{R}$, take a series of steps similar to those used in deriving equations (22). Suppose the notation so chosen that this leads to

where

$$
\begin{aligned}
& y_{2}=a_{n-2}\left(y_{1} ; x_{2}, \cdots, x_{n}\right), \\
& y_{3}=a_{n-3}\left(y_{1} ; x_{2}, \cdots, x_{n}\right) \text {, } \\
& y_{n}=a_{0}\left(y_{1}, x_{2}, \cdots, x_{n}\right) \text {, } \\
& x_{1}=f_{1}\left(y_{1}, a_{n-2}, \cdots, a_{0}\right)=F\left(x_{2}, \cdots, x_{n} ; y_{1}\right) \text {, }
\end{aligned}
$$

$$
\begin{gathered}
F(0, \cdots, 0)=F_{y_{1}}(0, \cdots, 0 ; 0)=\cdots=F_{\nu_{1}^{k-1}}(0, \cdots, 0 ; 0)=0, \\
F_{y_{1}}(0, \cdots, 0 ; 0) \neq 0 .
\end{gathered}
$$

The Weierstrassian preparation theorem puts in evidence the $k$-fold root $y_{1}$ of $F-x_{1}=0$, a continuous function of $x_{1}, \cdots, x_{n}$ which satisfies the conditions of Theorem 5. If the Weierstrassian polynomial has a discriminant not identically zero, its root will have $k$ distinct values in any point not on the locus defined by equating to zero the discriminant. But this discriminant is equivalent to the result of eliminating $y_{1}$ from $F\left(x_{2}, \cdots, x_{n} ; y_{1}\right)-x_{1}$ and $F_{y_{1}}-0$, and therefore can not be zero for all values of $\left(x_{1}, \cdots, x_{n}\right)$. For the necessary and sufficient condition that $F-x_{1}$ and $F_{y_{1}}$ admit a common factor throughout the region in which they are defined is that the discriminant vanish identically. Since $F$ does not involve $x_{1}$, the function $F-x_{1}$ is irreducible and is not a factor of $F_{y_{1}}$. Therefore the discriminant is not identically zero, and except at points for which this discriminant is zero the solution $y_{1}$ of $F-x_{1}=0$ has $k$ distinct values; consequently in this point equations (25) have an analytic inverse with $k$ distinct branches. Further, the discriminant, while not identically zero, is always zero at the origin, and hence is zero along a definite locus through the origin. Along this locus the continuous solution found for equations (25) is less than $k$ valued. Since $F-x_{1}$ is irreducible, $y_{1}$ is a single $k$-valued function of $x_{1}, \cdots, x_{n}$, which can not be broken up into two or more functions each with a lower order of multiplicity. Therefore, if $k>1$, the inverse transformation is defined by functions everywhere continuous, but not everywhere analytic. This completes the proof of Theorem 5.

UNIVERSITY OF WISCONSIN, Madison, Wisconsin. 